\title{
Scénariser une situation d'apprentissage collective instrumentée : réalités, méthodes et modèles, quelques pistes
}

Jean Pierre David

Laboratoire Informatique de Grenoble, FRANCE

Jean-Pierre.David@imag.fr

Sébastien George

Laboratoire LIESP, INSA - Lyon, FRANCE

Sebastien.George@insa-lyon.fr

\section{Hélène Godinet}

Équipe EducTice, Institut national de recherche pédagogique, Lyon, FRANCE helene.godinet@inrp.fr

\section{Emmanuelle Villiot-Leclercq}

Laboratoire Informatique de Grenoble, FRANCE

emmanuelle.villiot-leclercq@imag.fr

Scénariser, une activité de formation

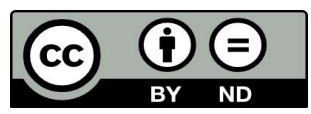

(C)Auteur(s). Cette œuvre, disponible à http://www.ritpu.org/IMG/pdf/ritpu0402_david.pdf, est mise à disposition selon les termes de la licence Creative Commons Attribution - Pas de Modification 2.5 Canada : http://creativecommons.org/licences/by-nd/2.5/ca/deed.fr
\end{abstract}

\section{Résumé}

Le projet de recherche ACTEURS (Activités collectives et tutorat dans l'enseignement universitaire : réalités, scénarios) s'intéresse aux pratiques des acteurs dans le cadre de dispositifs d'apprentissage à distance ou hybrides dans l'enseignement supérieur, ainsi qu'aux artefacts employés. Le projet s'est donné pour objet de définir et analyser des situations d'apprentissage collectives instrumentées (SACI) au sein de dispositifs de formation. La question de la scénarisation des SACI s'inscrit dans une démarche de réutilisation et de mutualisation de pratiques rendues possibles par l'usage de plateformes de téléenseignement. Notre étude a permis de caractériser les SACI et de repérer le besoin de scénariser la collaboration. Nous proposons une approche itérative pour exprimer et réutiliser un scénario de SACI. Nous utilisons tout d'abord un scénario type pour un type d'activités pédagogiques spécifiques. Ensuite, nous l'exprimons selon le formalisme des Pléiades qui permet la traduction du scénario dans un langage de modélisation pédagogique pour aboutir à une proposition d'opérationnalisation dans un environnement informatique d'apprentissage.

\section{Abstract}

The ACTEURS research project (Collective activities and tutoring in higher education: Realities, scenarios) has focused on the actors' practices within the framework of distance or hybrid learning environments in higher education, as well as in the use of artefacts. It aims at defining and analysing instrumented collective learning situations (SACI). The question of SACI scenarising takes place in a process of reuse and mutualization of practices, made possible by the use of telelearning platforms. Our study allows to characterize the SACI and to track down the need of scenarising the collaboration. We propose an iterative approach to express and reuse a scenario of SACI. First of all, we use a scenario-type for specific educational activities. Then we express it according to the Pleiades formalism which allows the translation of the scenario in an educational modelling language to implement it on a telelearning platform. 


\section{Introduction}

Le projet de recherche ACTEURS (Activités collectives et tutorat dans l'enseignement universitaire : réalités, scénarios) réunit des équipes de recherche de différentes disciplines (informatique, psychologie, ergonomie, sociologie, sciences de l'éducation, de la communication, de la cognition et du langage) de la région Rhône-Alpes. La recherche ACTEURS, organisée en trois pans, s'intéresse aux pratiques des acteurs dans le cadre de dispositifs d'apprentissage à distance ou hybrides dans l'enseignement supérieur, ainsi qu'aux artefacts employés. Un pan du projet, dont il est question dans cet exposé, s'est donné pour objet de définir et analyser des situations d'apprentissage collectives instrumentées (SACI) au sein de dispositifs de formation.

Nous définissons une SACI comme étant

une situation pédagogique avec un objectif d'apprentissage (en termes de connaissances et/ou de compétences), des acteurs identifiés, une durée et un mode d'évaluation des apprenants. Elle prend la forme d'une unité d'apprentissage scénarisée dans laquelle la production individuelle et/ou collective attendue est liée à une activité collective instrumentée par des artefacts informatiques (Bourriquen et al., 2006).

L'étude d'une douzaine de SACI sur des terrains de formation francophones (campus numériques, FOAD, formations hybrides, etc.) a permis de dégager un certain nombre d'éléments de caractérisation des SACI et de révéler la complexité de leurs mises en œuvre effectives. La question de la scénarisation des SACI, présentée ici, s'inscrit dans une démarche de réutilisation de ressources pédagogiques et/ou de mutualisation de pratiques que rend possible l'usage de plateformes de téléenseignement.

Notre étude a permis d'une part de caractériser une SACI, d'autre part de repérer le besoin de scénariser les situations collaboratives. Nous proposons une approche pour expri- mer un scénario de SACI potentiellement réutilisable. Une première phase correspond à ce qu'on appelle ici un scénario type, exprimé "à la main », utilisé et réutilisé pour un type d'activités pédagogiques spécifiques; une deuxième phase propose une méthode de scénarisation, la méthode des Pléiades, qui permet la traduction du scénario dans un langage de modélisation pédagogique à la sémantique proche de celles des enseignants; la dernière phase pose les jalons d'une opérationnalisation d'un scénario de SACI dans un environnement informatique d'apprentissage.

\section{Structure hiérarchique d'une SACI}

Pour décrire une SACI, nous utilisons un certain nombre de concepts issus de la théorie de l'activité. En particulier, nous nous appuyons sur une structure hiérarchique articulant trois niveaux d'activité proposée dans Leontiev (1981) : les intentions, les actions et les opérations. Au niveau supérieur, l'intention est dirigée et motivée par un objet. Au niveau intermédiaire, les actions sont dirigées par des buts. Au niveau inférieur, nous trouvons les opérations qui n'ont pas de buts propres, mais sont soumises à des conditions.

Nous utilisons cette structure hiérarchique d'une activité pour éclairer les caractéristiques d'une SACI (figure 1). Nous proposons d'ajouter un niveau transversal (ou englobant) qui précise le contexte global d'une formation. Les objectifs pédagogiques (apprendre à collaborer, objectif disciplinaire...), le type de public visé (primaire, secondaire, supérieur) et les modalités spatiales et temporelles de réalisation de la formation (présence, distance, hybride) font ainsi partie du contexte. Si nous avons choisi de placer les objectifs pédagogiques à ce niveau, c'est parce que ces éléments conditionnent le type d'activité pédagogique collective à mettre en place. Par exemple, un débat, une résolution de problème ou une étude de cas sont des types d'activité qui peuvent se décliner sous forme de scénarios d'activités plus précis. Au niveau intermédiaire, nous retrouvons les actions qui sont réalisées dans le cadre d'une activité. Ces actions ont des caractéristiques : la détermi- 


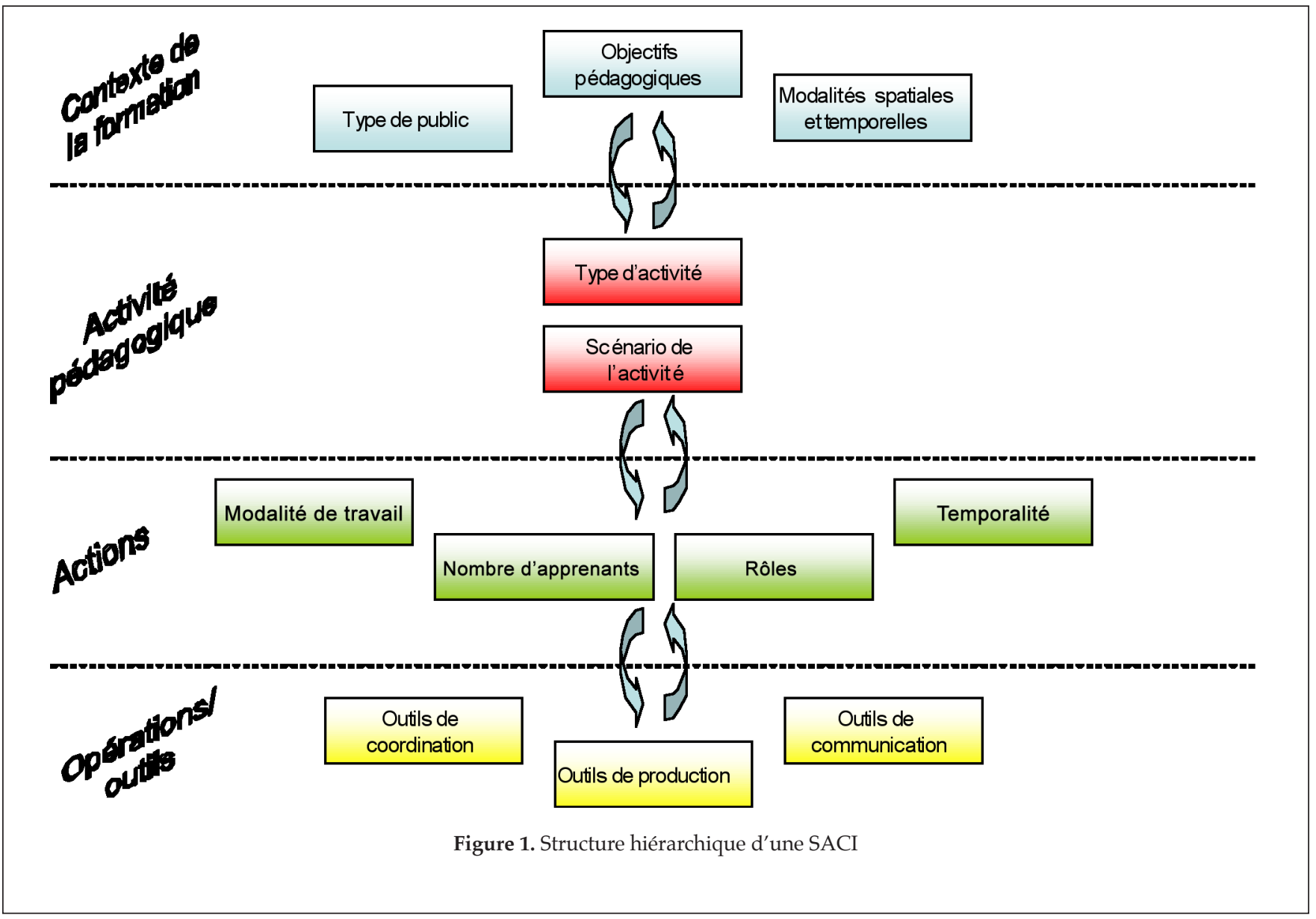

nation du nombre d'apprenants par groupe collectif et des rôles joués, la temporalité (synchrone, asynchrone) et la modalité de travail (individuel, coopération, collaboration, compétition...). Enfin, le niveau inférieur concerne les opérations qui, dans le cas d'une activité médiatisée, se réalisent avec des outils ou artefacts : outils de production (éditeur de texte, tableau blanc...), outils de communication (courriel, chat, forum...) et outils de gestion et de coordination (agenda, gestion de la documentation...).

Les différents niveaux décrits à la figure 1 se répondent dans une relation dynamique. Les niveaux sont mutuellement conditionnés ou influencés. Par exemple, le type de public et les objectifs pédagogiques visés guideront la mise en place d'une activité pédagogique. Cette même activité conditionnera les actions à réaliser, certaines d'entre elles nécessitant des outils informatiques (actions médiatisées).
Nous retrouvons ainsi la relation duale existant entre activité et outil : 1'activité conditionne les outils et la manière de les utiliser, et, en retour, les outils structurent l'activité des utilisateurs. Ce processus de genèse instrumentale (Rabardel, 1995) explique comment les outils contraignent l'activité collective, sans la déterminer complètement, car les outils sont toujours utilisés en situation.

Dans le cas d'une SACI, il est intéressant d'observer si et comment ses caractéristiques sont influencées/conditionnées par la structure hiérarchique identifiée. Nous avons pu observer dans la majorité des SACI étudiées qu'un écart existait entre les activités prescrites par les concepteurs pédagogiques et leur déroulement effectif. Cet écart entre la tâche prescrite et l'activité effectivement réalisée peut-il s'expliquer par un choix inadéquat entre l'activité à mettre en place et les outils proposés? Ce type d'écart peut être constaté tant au niveau des actions qu'au niveau des 
opérations/outils. D'une part, les actions sont bien plus souvent individuelles que collaboratives ou coopératives comme escompté par les concepteurs, d'autre part, l'utilisation effective des outils du dispositif technique est généralement en deçà des attentes, ceci dans les situations que nous avons pu observer (Michel, Garrot et George, 2007).

En référence aux approches IMS-LD proposées par Koper et Tattersall (2005), nous faisons l'hypothèse que la scénarisation des activités pourrait tenir un rôle central dans le déroulement d'une SACI. Dans les observations effectuées auprès de 12 terrains de SACI, un scénario existe pour $80 \%$ des SACI étudiées. Cependant, bien qu'il soit déclaré comme existant, ce scénario est rarement explicité de façon détaillée ou selon un formalisme qui en faciliterait 1'appropriation et la mise en œuvre par des acteurs autres que le concepteur initial. Dans la plupart des cas, la présentation du scénario de SACI se limite à quelques lignes de texte. Nous faisons l'hypothèse que ce manque de scénarisation formelle pourrait être en partie à l'origine des écarts constatés entre la prescription d'activités collectives et leur réalité effective sur le terrain.

Pour la grande majorité des SACI observées, un double objectif pédagogique est explicitement formulé par les enseignants-concepteurs de la situation d'apprentissage : il s'agit tout à la fois de « collaborer pour apprendre » et d' " apprendre à collaborer ». Comment passer de l'intention pédagogique à l'action (ici, mettre les apprenants en situation de collaborer en leur proposant une situation d'apprentissage ad hoc)? Notre travail s'oriente donc vers une proposition d'aide à la scénarisation de SACI. Cet exposé propose divers niveaux de scénarisation, selon les trois approches qui suivent.

\section{Concevoir un scénario type réutilisable : le} scénario « étude de cas » du campus numérique FORSE (formation en sciences de l'éducation) ${ }^{1}$

\section{Situation collaborative type et canevas d'aide à la conception}

Les activités collaboratives nécessitent le partage des ressources, la négociation en vue de parvenir à un consensus et la production de documents de synthèse, ceci pour traiter une question complexe. Elles exigent de la part des acteurs un engagement à la fois explicite (chacun doit s'inscrire dans un rôle et contribuer) et organisé (le travail collaboratif n'est pas la somme de travaux individuels) (Godinet, 2007). Dans le campus numérique FORSE, pour expliciter et organiser les activités des différents acteurs (enseignants-auteurs, tuteurs, étudiants, évaluateurs), un scénario type est mis à disposition dans un espace dédié sur la plateforme. Ce scénario contraint

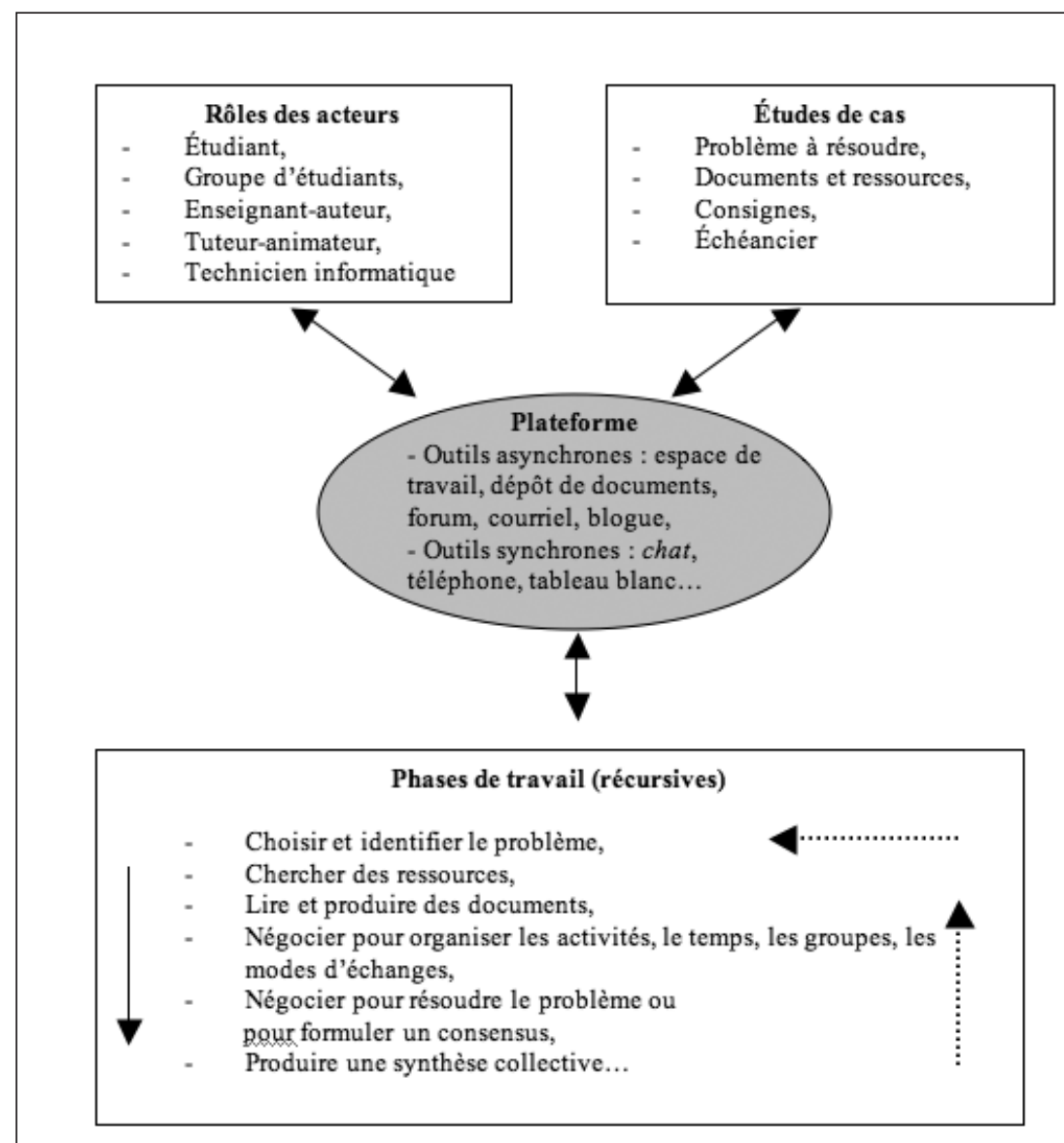

Figure 2. Modèle du scénario collaboratif «étude de cas » 


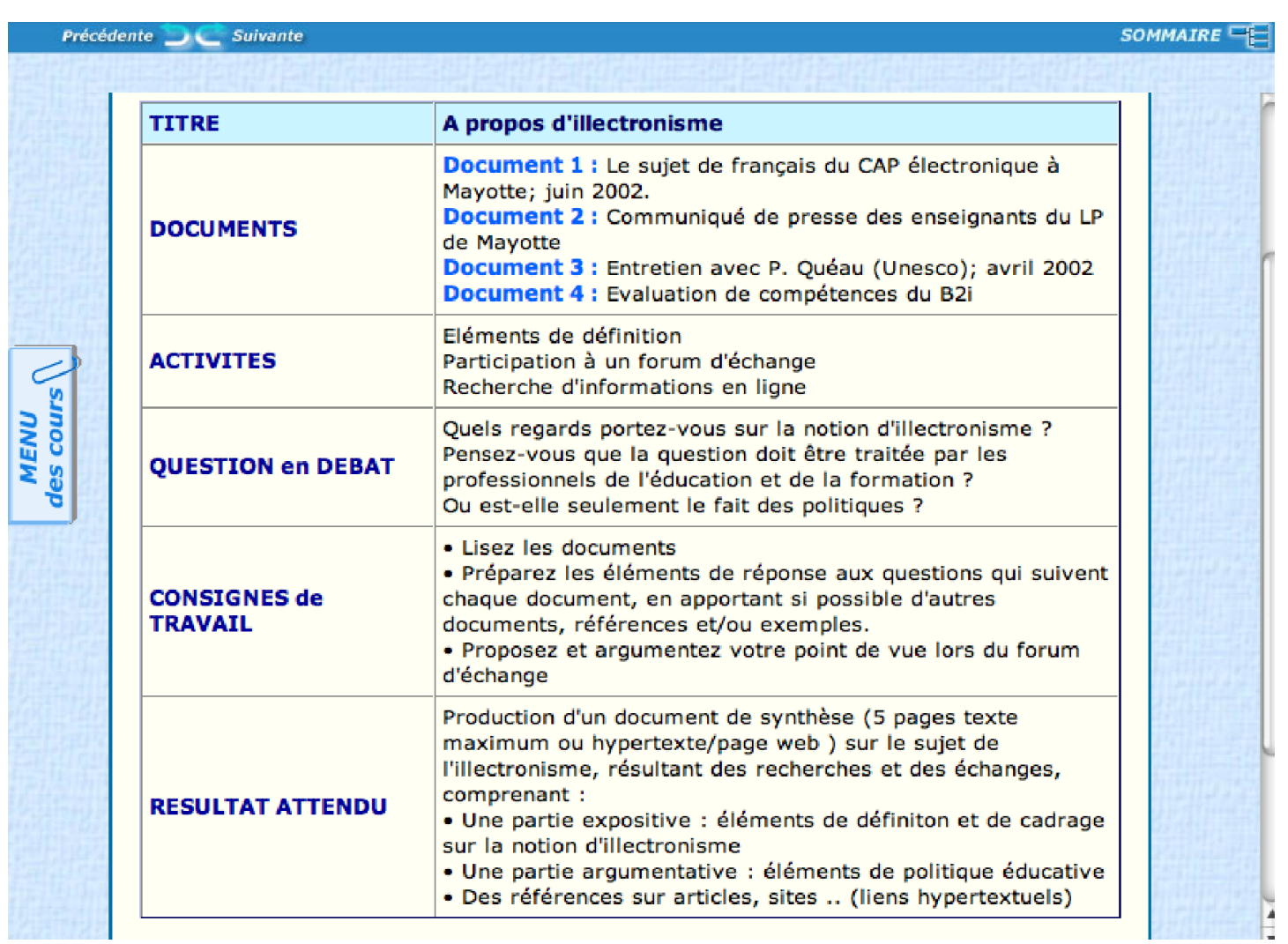

Figure 3. Un exemple de canevas de création d'étude de cas complété par l'enseignant-auteur

et invite à la fois les étudiants à la collaboration, en privilégiant le développement de compétences langagières et relationnelles. Le scénario s'appuie sur la dynamique interactionnelle des acteurs, des outils de communication et des ressources disponibles, comme le montre la figure 2.

À partir de ce scénario type, les acteurs peuvent jouer les variables de la situation : faire varier la temporalité des actions (date et durée des tâches proposées), le nombre et le type de ressources proposées (cours, documents d'aide, consignes, références bibliographiques et sitographiques, etc.), le nombre et le type de productions attendues (éléments de discussion du cas, éléments de méthode, documents intermédiaires, synthèses, etc.) et le nombre et les rôles des acteurs (attribution de telle ou telle tâche et/ou responsabilité à tel ou tel acteur ou groupe d'acteurs par rapport à l'état d'avancement et aux différentes phases du travail collectif, etc.). Le scénario est conçu de façon suffisamment flexible, d'une part pour être réutilisé dans des contextes proches (réutilisation du scénario en présentiel, réutilisation du scénario dans d'autres disciplines que les sciences de l'éducation, etc.), d'autre part pour être mis à disposition et éprouvé dans des environnements variés (plateforme de formation à distance, bureau virtuel, blogue pédagogique). Le scénario « étude de cas » est décrit de façon globale (figure 2) et se décline (lorsqu'il est interprété) sur un ensemble de canevas réutilisables (textes et tableaux disponibles dans des formats portables) : canevas de création de l'étude de cas pour l'enseignant-auteur (figure 3); canevas des activités de l'étudiant (consignes, échéancier, description d'usages des espaces dédiés à la production, la communication, etc.); canevas de l'encadrement pédagogique (figure 4), etc.

\section{Usage du scénario type par les différents acteurs}

Le scénario type guide l'enseignant-auteur à qui il est demandé de produire une étude de cas, ainsi que le tuteur à qui il est de- 


\begin{tabular}{|c|c|c|}
\hline Acteurs & Activités nécessaires & Activités possibles \\
\hline $\begin{array}{l}\text { Enseignant- } \\
\text { auteur- } \\
\text { concepteur }\end{array}$ & $\begin{array}{l}\text { - Production des études de cas } \\
\text { - Production des consignes } \\
\text { - Structuration temporelle de } \\
\text { l'activité } \\
\text { - Evaluation des productions } \\
\text { - Evaluation de la participation } \\
\text { - Evaluation de la collaboration }\end{array}$ & $\begin{array}{l}\text { - Mise en ligne } \\
\text { - Régulation (reformulation, } \\
\text { compléments d'information ...) } \\
\text { - Participation aux chats, forums }\end{array}$ \\
\hline $\begin{array}{l}\text { Animateur de } \\
\text { plateforme } \\
\text { ou tuteur }\end{array}$ & $\begin{array}{l}\text { - Gestion des groupes } \\
\text { - Régulation (rappel des } \\
\text { consignes, rappel des échéances, } \\
\text { encouragements, explicitations via } \\
\text { mail, forum, chat) } \\
\text { - Sauvegarde / classement des } \\
\text { productions }\end{array}$ & $\begin{array}{l}\text { - Regard critique sur les } \\
\text { productions } \\
\text { - Aide à l'évaluation } \\
\text { - Participation aux chats portant } \\
\text { sur chaque étude }\end{array}$ \\
\hline $\begin{array}{l}\text { Développeur } \\
\text { informatique }\end{array}$ & $\begin{array}{l}\text { - Informatisation des contenus de } \\
\text { cours, des canevas « études de } \\
\text { cas » } \\
\text { - Création d'espaces de travail sur } \\
\text { la plateforme } \\
\text { - Informations techniques à } \\
\text { destination des usagers }\end{array}$ & $\begin{array}{l}\text { - Création de fiches d'aide pour les } \\
\text { manipulations informatiques } \\
\text { - Aide technique en ligne } \\
\text { - Archivage des contenus des } \\
\text { mails, chats et forums }\end{array}$ \\
\hline
\end{tabular}

Figure 4. Canevas de l'encadrement pédagogique pour une SACI «étude de cas »

mandé d'en assurer le suivi, la régulation et/ou l'évaluation en ligne. Dès lors que les enseignants-auteurs adhèrent au modèle de l'activité « étude de cas », leur intervention dans la plateforme est à la fois contrainte (fortement guidée) et allégée par ce même guidage. Leur expertise, d'abord en ce qui concerne le domaine de contenu du cours (économie de l'éducation, philosophie de l'éducation, sociologie de l'éducation, etc.), ensuite en ce qui concerne la méthode d'appropriation des connaissances (recherche d'informations, compréhension, reformulation, vérification, restitution, etc.) et enfin en ce qui concerne la régulation des interactions (intercompréhension, négociation, construction de consensus...), peut être d'autant plus sollicitée que, parallèlement, leur implication dans l'orchestration des activités collectives, complexifiée par la distance et/ou les fonctionnalités de la plateforme, est fortement assistée par le scénario. Or, cette assistance à l'orchestration des activités est souhaitée par bon nombre d'enseignants du supérieur qui n'ont pas encore intégré tant les usages des technologies numériques que les pratiques d'apprentissage collaboratives à distance à leur culture professionnelle, ce qu'ont pu constater Develay, Ciekanski et Godinet (2006).

Les enquêtes conduites auprès de plusieurs cohortes d'étudiants usagers de ce scénario " étude de cas » ont montré que l'étudiant en ligne apprécie d'être fortement guidé dans les activités d'apprentissage qui nécessitent de collaborer à distance; si la collaboration est explicitement scriptée dans le scénario (rôles, phases), l'étudiant s'y engage parce qu'il peut évaluer a priori le rapport coût/bénéfice de son engagement (Baluteau et Godinet, 2006; Béziat, Godinet et Wallet, 2005).

Quelques pistes pour rendre la réutilisation $\mathrm{du}$ scénario plus opérationnelle

La difficulté de la scénarisation est de concevoir un modèle dynamique qui puisse à la fois rendre compte de la complexi- 
té d'une situation d'apprentissage. D'abord parce qu'on sait bien que l'activité d'enseigner comme celle d'apprendre sont particulièrement difficiles à décrire (Pastré, P., Mayen, P. et Vergnaud, G. (2006), ensuite parce que la nature de l'engagement des étudiants, dans une activité d'apprentissage collective ou non, est imprévisible alors même que cet engagement conditionne l'interprétation du scénario (Dufresne, 2001). Le scénario " étude de cas » est révélateur d'un processus de collaboration dans lequel le résultat (de cette collaboration) se construit en même temps que la communauté qui en est l'auteur.

La faiblesse de ce mode de scénarisation réside aussi dans sa relative portabilité. À partir du modèle (figure 2), chaque canevas est accessible isolément et doit être complété « à la main » par l'enseignant-auteur de la SACI, et ce, pour chacune des réutilisations (exemple, figure 3). Dans cet exemple de SACI, l'enseignant-auteur de l'étude de cas complète un canevas qui prescrit l'activité pédagogique (fichier au format texte) et y adjoint les éléments nécessaires (autres documents et ressources). L'administrateur de la plateforme se charge ensuite de formater ces divers documents, de faire des liens s'il y a lieu et de les mettre à disposition des acteurs (tuteurs, étudiants) dans le temps de l'activité. Si le scénario était formalisé de façon plus explicite, tous les éléments nécessaires à l'exécution de la SACI pourraient être orchestrés de façon moins artisanale. Ceci permettrait d'une part aux différents acteurs de jouer chacun leur rôle (par exemple, actualiser les variables de chacun des canevas; ou encore interagir à tel ou tel moment) et d'autre part de rendre visibles et cohérentes les différentes actions collectives (échanger, communiquer, produire des documents, les mettre à disposition, etc.). L'opérationnalisation du scénario (voir infra) dans un dispositif instrumenté permettrait d'agencer toute la SACI, de façon réticulaire et dynamique, en fonction du contexte pédagogique choisi (acteurs, niveau, discipline, temporalité, etc.).

Cet exemple d'usage du scénario type « étude de cas » illustre le besoin de décrire des SACI, de manière plus formalisée, ceci afin d'améliorer leur réutilisabilité au sein de communautés d'apprentissage. Le problème qui se pose est double :
1) comment exprimer les scénarios pédagogiques de SACI de façon suffisamment formalisée pour qu'ils soient partageables et réutilisables au sein d'une communauté d'apprentissage? 2) comment intégrer dans cette formalisation les spécificités de l'approche pédagogique choisie et les intentions pédagogiques des enseignants?

Pour tenter de répondre à ce besoin (formaliser et réutiliser des scénarios types), nous présentons une méthode d'expression formalisée de scénarios pédagogiques collaboratifs, la méthode des Pléiades, et nous l'illustrons à travers une situation collaborative de type "étude de cas ».

\section{Formaliser un scénario type réutilisable : l'approche par la méthode des Pléiades}

L'enjeu de cette recherche est de permettre aux enseignants d'exprimer de façon formalisée les SACI tout en leur donnant les moyens d'expliciter leurs choix pédagogiques sousjacents. La méthode des Pléiades (Villiot-Leclercq, 2007) vise à soutenir la conception des scénarios structurés et réutilisables en cherchant à réduire l'écart entre un formalisme générique, nécessaire au partage et à l'opérationnalisation des scénarios dans des situations d'apprentissage instrumentées, et les choix pédagogiques propres à chaque enseignant et à chaque contexte.

Cette méthode de scénarisation, qui prend appui sur les travaux en psychologie cognitive issus de la théorie de la forme, ou Gestalt-théorie (Guillaume, 1979), est le résultat d'une démarche de recherche et de développement centrée usagers impliquant des enseignants et des concepteurs pédagogiques (Villiot-Leclercq, sous presse).

Il existe actuellement des propositions pour formaliser les scénarios pédagogiques : MISA (méthode d'ingénierie des systèmes d'apprentissage) (Paquette, 2002) ou encore des langages de modélisation pédagogique, appelés aussi EML (educational modelling language) tels que LDL (learning design language) (Martel, Vignollet et Ferraris, 2006) et IMS Global Learning Consortium (2003). 
Chacune de ces propositions de formalisation a fait l'objet de nombreux travaux, ces dernières années, qui en soulignent les intérêts et les limites. Dans cet article, nous n'aborderons pas ces débats dans la mesure où la proposition de formalisme ne se présente pas «contre » les autres approches, mais comme une solution de rechange à l'expression de scénarios formalisés. Cette alternative repose sur l'hypothèse que la « mise en forme » que nécessite la démarche de formalisation ne doit pas se faire au détriment de l'intention pédagogique initiale mais que, au contraire, elle doit permettre la sauvegarde du sens de la situation d'apprentissage. Pour la construire, nous avons pris comme point de départ les attentes des enseignants quant au formalisme d'expression de scénarios pédagogiques. Un travail sur différents formalismes (Villiot-Leclercq, David et Lejeune, 2006) a permis, en amont, de préciser les critères auxquels un formalisme d'expression des scénarios devait répondre : facilité de structuration du scénario et de mise en évidence des activités principales et secondaires, facilité de mise en relation des différents aspects du scénario (ressources, activités, rôles, outils), possibilité d'expliciter les intentions et les choix pédagogiques. Ce premier travail a abouti à la proposition du formalisme des Pléiades.

\section{Principe et propriétés}

Ce formalisme se fonde sur le principe d'entité signifiante d'activités, qui repose sur la métaphore astronomique des Pléiades. Les Pléiades sont un regroupement d'étoiles identifiables visuellement dans le ciel que l'œil humain perçoit comme une forme cohérente. Cette forme, au regard des autres objets de l'espace, est perçue comme une entité à part entière. Même si la proximité n'est qu'apparente, l'œil et le cerveau reçoivent les informations, les interprètent et donnent un sens et un nom à cette forme. La perception et la réception sont un processus individuel dont le résultat (la forme et l'interprétation que l'on en fait) peut être partagé et peut constituer une interprétation collective, un «horizon d'attente » (Jauss, 1972). Dans le domaine de la scénarisation, le scénario pédagogique peut être défini comme le résultat écrit d'une intention individuelle ou collective ( $d$ 'un ou des enseignants) et d'une interprétation individuelle ou collective. En tant que résultat écrit, le scénario pédagogique vise à définir les activités, ressources et acteurs impliqués dans la situation d'apprentissage (Paquette, 2005), mais cette définition passe par l'expression d'un ensemble d'énoncés, un mot ou un groupe de mots qui constituent une entité de sens. Dans notre approche, une entité de sens se constitue par référence, soit à une activité d'apprentissage, soit à un regroupement d'activités auquel on peut attribuer une identité et qui fait sens par rapport aux choix et au contexte pédagogiques de l'enseignant-auteur.

Nous proposons ainsi qu'un scénario pédagogique puisse être construit selon différents niveaux d'activités imbriquées. Nous avons donc défini trois niveaux de granularité : 1) un scénario de type activité élémentaire est une étoile, 2) un regroupement de plusieurs activités est une pléiade, 3) un regroupement de plusieurs regroupements ou pléiades est une constellation. Chaque grain est défini comme une entité cohérente dont le sens se construit par rapport aux choix et aux intentions pédagogiques de l'enseignant-auteur et par l'interprétation qu'en fera l'enseignant-lecteur. Pour que ce processus de production du sens à l'écriture et à la lecture s'enclenche et débouche éventuellement sur une réutilisation, l'enseignant doit pouvoir prendre le rôle de l'énonciateur et dire, sous forme d'énoncés relativement courts et organisés, quels sont les paramètres de cette entité.

C'est ainsi que dans le formalisme des Pléiades, chaque entité (par exemple un regroupement d'activités qui vise à évaluer des apprenants au début d'une année scolaire - Pléiade «Évaluation diagnostique ») est associée à un ensemble de propriétés auxquelles il est possible d'attribuer des valeurs sous forme d'énoncés. Une partie des propriétés permet de décrire certaines caractéristiques de l'entité concernée : la granularité (par ex., pléiade), le nom (par ex., évaluation diagnostique), le statut dans le scénario (optionnel), les activités constituantes, les stratégies d'enseignement et d'apprentissage (stratégie individuelle/ activation des connaissances antérieures) et les éléments de connaissances. Ce sont des propriétés descriptives qui ont 


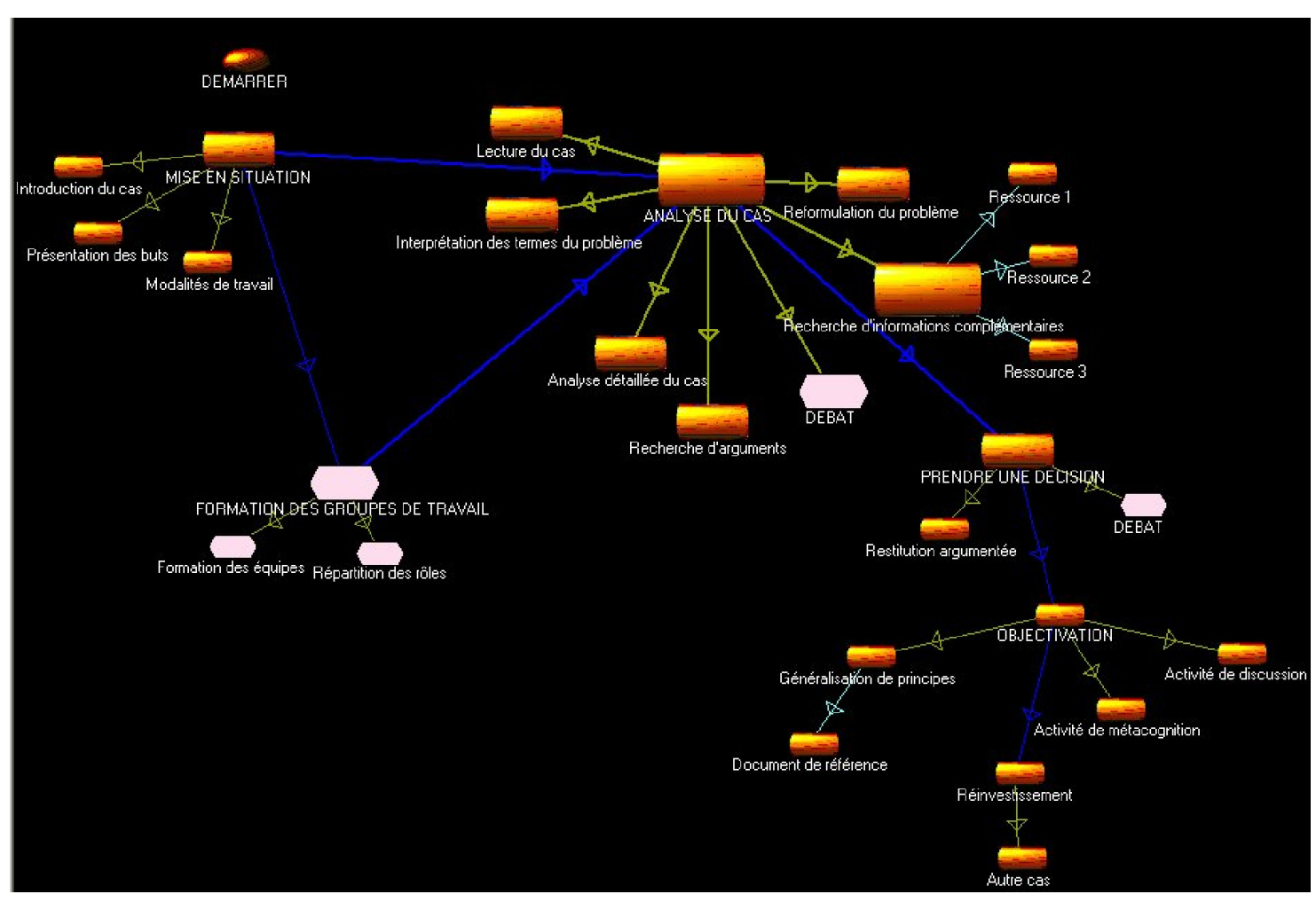

Figure 5. Patron de scénario « étude de cas » formalisé avec la méthode des Pléiades et opérationnalisé dans ExploraGraph (Dufresne, 2001)

valeur de métadonnées et qui peuvent se révéler importantes dans le processus de réutilisation lors de la recherche et de la sélection d'un scénario existant.

Une autre partie des propriétés, les propriétés organisatrices, permettent d'organiser l'entité de façon dynamique. Dans le cas où cette entité est un regroupement d'activités, l'enseignant peut, par le biais de ces propriétés, énoncer un ensemble de paramètres à lui appliquer : " l'orchestration » des activités (ordre, sans ordre, etc.), les « conditions de démarrage ", les « conditions de clôture » (choix de l'apprenant, choix de l'enseignant, contrainte temporelle), la « distribution » des activités entre les acteurs (élèves, enseignants, élèves-élèves, élèves-expert), ainsi que l'apport des éléments supports (ressources utilisées, ressources produites, outils utilisés).

Ces informations données sous forme d'énoncés, au choix des enseignants, ont pour buts de paramétrer cha- que grain constituant le scénario et, surtout, d'organiser leur enchaînement.

\section{Exemple : formalisation du scénario " étude de cas »}

Afin d'illustrer cette méthode, nous proposons de formaliser un scénario de type «étude de cas ». Pour dégager la spécificité de cette approche et justifier le choix de certains énoncés, nous nous référons aux travaux de Guilbert et Ouellet (2004) centrés sur l'étude de cas. Le scénario représenté est de grain « constellation », il regroupe différentes pléiades d'activités : mise en situation, formation des groupes de travail, analyse du cas, etc.

Chaque pléiade et chaque activité la constituant pourront être également définies par le même ensemble de propriétés. Ainsi, nous pourrons formaliser, de la même façon, l'entité "Mise en situation » en définissant les activités associées et leur orchestration, mais aussi la façon dont elles sont distribuées entre les différents acteurs impliqués. 
Tableau 1. Formalisation du patron « étude de cas » avec la méthode des Pléiades

\begin{tabular}{|c|c|}
\hline Propriétés & Valeurs qui peuvent être modifiées \\
\hline Granularité & Constellation \\
\hline Famille de regroupement & Étude de cas \\
\hline Statut & Obligatoire \\
\hline Stratégie d'apprentissage & Élaboration et organisation des connaissances \\
\hline Stratégie d'enseignement & Stratégie socioconstructiviste \\
\hline Densité & 6 \\
\hline Constituants & $\begin{array}{l}\text { 1. Mise en situation } \\
\text { 2. Formation des groupes de travail } \\
\text { 3. Analyse du cas } \\
\text { 4. Prise de décision } \\
\text { 5. Objectivation } \\
\text { 6. Réinvestissement }\end{array}$ \\
\hline Temps & $3 \times 2 h$ \\
\hline Orchestration & $\{1 ; 2 ; 3 ; 4 ; 5 ; 6\}$ séquentielle \\
\hline Conditions de clôture & $\begin{array}{l}\text { Par l'enseignant }\{1 ; 2 ; 5 ; 6\} \\
\text { Par l'élève }\{3 ; 4\}\end{array}$ \\
\hline Distribution & $\begin{array}{l}\text { Formule } 1 \text { : enseignant-élève }\{1 ; 2 ; 5\} \\
\text { Formule } 2 \text { : élèves-élèves }\{3 ; 4 ; 6\}\end{array}$ \\
\hline Éléments de connaissances & Le scénario porte sur le paysage industriel au XIXe siècle \\
\hline Éléments de support & $\begin{array}{l}\text { Ressources : trois ensembles de documents: } \\
\text { - La description du cas } \\
\text { - Dossier contenant des textes et des images } \\
\text { Outils : le forum Agora et la messagerie }\end{array}$ \\
\hline
\end{tabular}

L'apport de la formalisation par les Pléiades à la réutilisation de scénarios pédagogiques

Dans Villiot-Leclercq et David (2007), nous avions proposé d'envisager le processus de réutilisation d'un scénario pédagogique en trois temps : le choix, l'appropriation, l'adaptation (modèle de réutilisation CAA). En effet, les enjeux sont de permettre aux enseignants de :

- choisir, parmi l'ensemble de scénarios existants, celui qui semble le plus pertinent au regard d'un ensemble de variables contextuelles et de leurs intentions;

- s'approprier les scénarios existants en leur permettant de disposer d'un scénario structuré et formalisé, et de s'appuyer sur un langage connu, voire partagé par leur communauté de praticiens (enseignants) ou leur communauté disciplinaire;
- donner la possibilité d'adapter facilement le scénario choisi. En ce sens, l'enseignant doit avoir la possibilité d'utiliser des scénarios types.

La méthode des Pléiades vise à répondre en partie à ces trois temps du processus de réutilisation. Elle offre une façon de structurer par des énoncés ou des groupements d'énoncés, au sein d'entités identifiées, ce que l'enseignant a souhaité mettre en œuvre et d'en partager la compréhension. Cette structuration par grains permet en outre à un enseignant, au sein d'une communauté de pratique, de rendre lisible son scénario et de le soumettre à une autre interprétation et à une réutilisation complète ou partielle dans un autre contexte. Dans le cas où l'interprétation des énoncés est partagée et semble correspondre à un horizon d'attente commun (disciplinaire, culturel, thématique, etc.), ce formalisme permet de dégager des invariants et d'identifier des patrons adaptables. Enfin, les propriétés descriptives 
peuvent soutenir les démarches de recherche et de choix de scénarios réutilisables au sein de banques de scénarios.

En définitive, la méthode des Pléiades vise à soutenir la création et la réutilisation de scénarios en proposant un mode d'expression formel tout en restant proche, sur les plans sémantique et structurel, des connaissances et des habitus des enseignants. Les premières évaluations du formalisme ont mis en évidence ses limites, mais aussi sa capacité à créer une zone de familiarité entre l'expression formelle du scénario, nécessaire pour envisager l'instrumentation et l'opérationnalisation totale ou partielle d'une SACI dans un contexte d'apprentissage instrumenté, et les intentions pédagogiques des enseignants.

\section{Que veut dire opérationnaliser un scénario collaboratif sur une plateforme?}

Quand un scénario de SACI a été conçu, il faut envisager la mise en œuvre, sur un environnement numérique de travail (ENT), de la situation d'apprentissage concernée, avec les différents acteurs impliqués dans le scénario. C'est la phase d'opérationnalisation du scénario. Lorsque l'environnement avec les outils nécessaires est simplement mis à la disposition des acteurs, en leur laissant la responsabilité d'effectuer leurs activités conformément au scénario qui leur est transmis textuellement, on parlera d'opérationnalisation partielle. Lorsque l'environnement prend en charge l'exécution du scénario, en contrôlant l'enchaînement des phases et la collaboration des acteurs, on parlera d'opérationnalisation totale.

\section{Opérationnalisation partielle}

Avec un ENT classique, opérationnaliser partiellement un scénario collaboratif revient à effectuer un certain ensemble de manipulations sur la plateforme avec le rôle d'administrateur, depuis l'inscription des acteurs dans des groupes de discussion jusqu'à la transmission à chacun du but et des consignes pour l'activité, la définition d'un calendrier de travail, la mise à disposition des ressources et la supervision du déroulement avec les moyens offerts par l'ENT. C'est cette manière de procéder qui est décrite plus haut dans l'exemple du campus numérique FORSE, où le scénario est instancié sur une plateforme WebCT par l'intermédiaire d'un administrateur, d'après les consignes d'un enseignant-auteur, formulées dans un fichier texte.

\section{Opérationnalisation à l'aide de SCORM}

De nombreuses plateformes permettent l'agrégation d'objets pédagogiques dans des parcours selon la norme SCORM. Dans ce cas, si on peut parler d'opérationnalisation totale, elle se limite au déploiement de scénarios d'apprentissage individuel, SCORM ne prenant pas en charge les dimensions collaboratives. En effet, les espaces collaboratifs sont définis indépendamment des parcours. Ils sont présents mais ne peuvent être intégrés dynamiquement. Pour atteindre l'objectif de scénariser la collaboration, il est nécessaire de scénariser aussi l'usage des services collaboratifs de la plateforme pour traduire complètement le scénario d'activité qui exprime l'intention pédagogique.

\section{Opérationnalisation totale avec un langage de modélisation}

Les étapes de conception décrites dans les paragraphes précédents produisent, avec la méthode des Pléiades, une expression formalisée. Pour opérationnaliser cette expression formalisée directement sur un ENT donné, il faudrait disposer d'un « lecteur Pléiade » capable d'exécuter le scénario en orchestrant les activités des acteurs sur les outils de l'ENT. Pour l'instant, il nous a semblé plus réaliste, voire plus efficace, de passer par un langage de modélisation pédagogique pour lequel il existe déjà des lecteurs permettant de déployer des scénarios sur des ENT différents.

Parmi les choix possibles actuellement, nous avons surtout étudié le langage IMS-LD, issu du langage EML de l'Open University des Pays-Bas, ainsi que le langage LDL conçu par une équipe de l'Université de Savoie (Martel, Vignollet, Ferraris et Durand, 2006). Dans le cas d'IMS-LD, le lecteur est réalisé avec le système Coppercore. Dans le cas de LDL, une transformation automatique du scénario XML est faite via le LDI (learning design infrastructure), qui fournit un lecteur sur une plateforme spécifique ${ }^{3}$.

Nous préconisons de traduire un scénario Pléiade, pour l'instant manuellement, dans un langage de modélisation pédagogique. Une fois le scénario traduit, la dernière étape d'opérationnalisation consiste à implanter ce scénario via Coppercore ou LDI, sur une plateforme capable de le jouer. Dans les deux cas, une fois 
attribués aux acteurs inscrits sur la plateforme les rôles prévus dans le scénario, l'activité correspondant à ce scénario peut être lancée. Chaque acteur qui se connecte est alors sollicité pour jouer le rôle qui lui a été attribué et les interactions entre les acteurs s'enchaînent en fonction du scénario.

\section{Expérience de scénarisation et d'opérationnalisation de jeu collaboratif}

Nous pouvons citer le défi proposé à des équipes de recherche du domaine de la scénarisation pédagogique lors d'un atelier de la conférence ICALT 2006 : il s'agissait de scénariser le « jeu des planètes » (Vignollet, David, Ferraris, Martel et Lejeune, 2006). Pour chaque apprenant, le but du jeu était de reconstituer l'ordre des planètes du système solaire en collectant des indices complémentaires fournis à deux équipes différentes. Les équipes étaient créées dynamiquement au cours de la partie, chacune collaborant dans un espace propre, et tous les joueurs se questionnant dans un autre espace collaboratif. Les neuf participants de l'atelier ont présenté leur analyse et des éléments de conception du scénario. Seule la solution exprimée en LDL a pu être entièrement opérationnalisée sur une plateforme pour répondre à toutes les exigences du défi et a pu être démontrée au cours de l'atelier (Martel, Vignollet et Ferraris, 2006) . Cette confrontation a permis de comparer langages, méthodes et outils, et a fourni la preuve que le langage LDL et son lecteur LDI permettent d'envisager une solution pour une opérationnalisation complète d'un scénario fortement collaboratif sur une plateforme. Notre ambition est d'obtenir une telle opérationnalisation, après avoir modélisé des scénarios caractéristiques des SACI par la méthode des Pléiades.

\section{Conclusion et perspectives}

Partant du constat que les situations de collaboration effectives dans le téléenseignement sont en deçà des attentes des enseignants-concepteurs de ces situations d'apprentissage, nous avons fait l'hypothèse qu'une scénarisation plus explicite pouvait y remédier. Nous avons proposé d'une part la méthode des Pléiades pour aider à décrire formellement un scénario, d'autre part des pistes pour opérationnaliser un scénario d'apprentissage collaboratif sur une plateforme.
Dans la suite de notre projet, nous nous proposons de caractériser des scénarios d'activités qui favorisent l'émergence de collaborations effectives dans des contextes d'apprentissage. Pour y parvenir, nous allons dans un premier temps proposer une typologie de SACI avant de définir des modèles de scénario par type de SACI (étude de cas, débat, jeu, etc.). À partir de ces modèles, nous allons concevoir et modéliser quelques scénarios pour les SACI existantes. Notre équipe de projet, après avoir conçu ces scénarios caractéristiques dans une approche interdisciplinaire (informatique, sciences cognitives et sciences de l'éducation), pourra d'une part expérimenter leur déroulement en milieu écologique, d'autre part mesurer les effets de cette scénarisation relativement aux objectifs « collaborer pour apprendre » et « apprendre à collaborer », objectifs que les enseignantsconcepteurs de SACI expriment comme des compétences qu'ils souhaitent développer dans l'enseignement supérieur.

\section{Références}

Baluteau, F. et Godinet, H. (2006). Cours en ligne à l'université [CELU]. Usages des liens hypertextuels et curriculum connexe (rapport de recherche). Lyon : Institut national de recherche pédagogique [INRP]. Récupéré du site de l'Institut, section Publications Éditions électroniques : http:/ / www.inrp.fr/publications / editionelectronique

Béziat, J., Godinet, H. et Wallet, J. (2005, décembre). Le cyber-étudiant en sciences de l'éducation : un "modèle" en évolution? Communication présentée au colloque du Séminaire sur l'industrialisation de la formation [SIF]. Les institutions éducatives face au numérique, Paris, France.

Bourriquen, B., David, J.-P., Garrot, E., George, S., Godinet, H., Medélez, E. et al. (2006, avril). Caractérisation des situations d'apprentissage collectives et instrumentées dans le supérieur. Communication présentée à la 8e Biennale de l'éducation et de la formation, Lyon, France.

Develay, M., Ciekanski, M. et Godinet, H. (2006). Pour une écologie de la responsabilité en e-formation. Distances et savoirs, 4(1), 61-72.

Dufresne, A. (2001). Modèles et outils pour définir le soutien dans les environnements hypermédias d'apprentissage. Dans E. de Vries, J.-P. Pernin et J.-P. Peyrin (dir.), Hypermédias et apprentissages. Actes du cinquième colloque (p. 13-24). Lyon, France : Institut national de recherche pédagogique [INRP]. 
Godinet, H. (2007). Scénario pour apprendre en collaborant à distance : contraintes et complexité. Dans J. Wallet (dir.), Le campus numérique FORSE : analyses et témoignages (p. 113-132). Mont Saint-Aignan : Publications des Universités de Rouen et du Havre.

Guilbert, L. et Ouellet, L. (2004). Étude de cas et apprentissage par problème. Sainte-Foy, Canada : Presses de l’Université du Québec.

Guillaume, P. (1979). La psychologie de la forme. Paris : Flammarion.

IMS Global Learning Consortium. (2003). IMS learning design information model - version 1.0. Récupéré le 17 mars 2008 du site du consortium : http:/ / www.imsglobal.org/learningdesign/ldv1p0/ imsld_infov1p0.html

Jauss, H. R. (1972). Pour une esthétique de la réception. Paris : Gallimard.

Koper, R. et Tattersall, T. (dir.). (2005). Learning design: A handbook on modelling and delivering networked education and training. Berlin/ Heidelberg : Springer-Verlag.

Leontiev, A. N. (1981). The problem of activity in psychology. Dans J. Wertsch (dir.), The concept of activity in Soviet psychology (p. 37-71). Armonk, NY : Sharpe.

Martel, C., Vignollet, L. et Ferraris, C. (2006). Modeling the case study with LDL and implementing it with LDI. Dans Proceedings of the Sixth IEEE International Conference on Advanced Learning Technologies (p. 1158-1159). Washington, DC : IEEE Computer Society.

Martel, C., Vignollet, L., Ferraris, C. et Durand, G. (2006). LDL: A language to model collaborative learning activities. Dans E. Pearson et P. Bohman (dir.), Proceedings of World Conference on Educational Multimedia, Hypermedia and Telecommunications (EDMEDIA) 2006 (p. 838-844). Chesapeake, VA : Association for the Advancement of Computing in Education.

Michel, C., Garrot, E. et George, S. (2007). Instrumented collective learning situations (ICLS): The gap between theoretical research and observed practices. Dans C. Crawford, A. D. Williams, R. Carlsen et al. (dir.), Proceedings of Society for Information Technology and Teacher Education (SITE) 2007 (p. 895-901). Norfolk, VA : Association for the Advancement of Computing in Education.

Paquette, G. (2002). L'ingénierie du téléapprentissage : pour construire l'apprentissage en réseaux. Sainte-Foy, Canada : Presses de l’Université du Québec.

Paquette, G. (2005). Apprentissage sur Internet : des plateformes aux portails d'objets à base de connaissance. Dans S. Pierre (dir.), Innovations et tendances en technologies de formation et d'apprentissage (p. 1-30). Montréal : Presses Internationales Polytechnique.

Pastré, P., Mayen, P.et Vergnaud, G. (2006). La didactique professionnelle.
Revue française de pédagogie, 154, 145-198.

Rabardel, P. (1995). Les hommes et les technologies. Approche cognitive des instruments contemporains. Paris : Armand Colin.

Vignollet, L., David, J.-P., Ferraris, C., Martel, C. et Lejeune, A. (2006). Learning design of collaborative learning activities: Languages, models, and tools. Dans Proceedings of the Sixth IEEE International Conference on Advanced Learning Technologies (p. 1149-1151). Washington, DC : IEEE Computer Society.

Villiot-Leclercq, E. (2007). Modèle de soutien pour l'élaboration et la réutilisation de scénarios pédagogiques. Thèse de doctorat non publiée, Université Joseph Fourrier (Grenoble 1)/Université de Montréal, France/Canada.

Villiot-Leclercq, E. (sous presse). La méthode des Pléiades : un formalisme pour favoriser la transférabilité et l'instrumentation des scénarios pédagogiques. Sciences et technologies de l'information et de la communication pour l'éducation et la formation.

Villiot-Leclercq, E. et David, J.-P. (2007). Le formalisme des Pléiades pour la conception et l'adaptation de patrons de scénarios pédagogiques. Dans T. Nodenot, J. Wallet et E. Fernandes (dir.), Actes de la conférence Environnement informatique pour l'apprentissage humain (EIAH 2007) (p. 269-274). Paris/Lyon : Association des technologies de l'information pour l'éducation et la formation [ATIEF] et Institut national de recherche pédagogique [INRP].

Villiot-Leclercq, E., David, J.-P. et Lejeune, A. (2006). Expressing learning scenarios with computer independent models. Dans Proceedings of the Sixth IEEE International Conference on Advanced Learning Technologies (p. 520-522). Washington, DC : IEEE Computer Society.

\section{Remerciements}

Ce travail a été effectué dans le cadre du projet ACTEURS (2005-2007), financé par le Ministère de l'Éducation nationale et de la Recherche (ACI «Terrains, techniques, théories »).

\section{Notes}

1 Campus numérique FORSE (FOrmation Ressources en Sciences de l'Éducation), http: / / www.sciencedu.org

2 Installation nécessaire : PostgreSQL 7.3, serveur Microsoft SQL, Xerces pour Java 2.6.0, JBoss 3.2.1.

3 La plateforme d'instrumentation est développée sur Plone par la société Pentila (Martel, Vignollet, Ferraris et Durand, 2006. 